\title{
How superfluid vortex knots untie
}

\author{
Dustin Kleckner ${ }^{1 \star \dagger}$, Louis H. Kauffman ${ }^{2}$ and William T. M. Irvine ${ }^{1 \star}$
}

Knots and links often occur in physical systems, including shaken strands of rope ${ }^{1}$ and DNA (ref. 2), as well as the more subtle structure of vortices in fluids ${ }^{3}$ and magnetic fields in plasmas ${ }^{4}$. Theories of fluid flows without dissipation predict these tangled structures persist ${ }^{5}$, constraining the evolution of the flow much like a knot tied in a shoelace. This constraint gives rise to a conserved quantity known as helicity ${ }^{6,7}$, offering both fundamental insights and enticing possibilities for controlling complex flows. However, even small amounts of dissipation allow knots to untie by means of 'cutand-splice' operations known as reconnections ${ }^{3,4,8-11}$. Despite the potentially fundamental role of these reconnections in understanding helicity - and the stability of knotted fields more generally-their effect is known only for a handful of simple knots $^{12}$. Here we study the evolution of 322 elemental knots and links in the Gross-Pitaevskii model for a superfluid, and find that they universally untie. We observe that the centreline helicity is partially preserved even as the knots untie, a remnant of the perfect helicity conservation predicted for idealized fluids. Moreover, we find that the topological pathways of untying knots have simple descriptions in terms of minimal two-dimensional knot diagrams, and tend to concentrate in states which are twisted in only one direction. These results have direct analogies to previous studies of simple knots in several systems, including DNA recombination ${ }^{2}$ and classical fluids $^{3,12}$. This similarity in the geometric and topological evolution suggests there are universal aspects in the behaviour of knots in dissipative fields.

Tying a knot has long been a metaphor for creating stability, and for good reason: untangling even a common knotted string requires either scissors or a complicated series of moves. This persistence has important consequences for filamentous physical structures such as DNA, the behaviour of which is altered by knots and links ${ }^{9,13}$. An analogous effect can be seen in physical fields, for example, magnetic fields in plasmas or vortices in fluid flow; in both cases knots never untie in idealized models, giving rise to new conserved quantities ${ }^{6,14}$. At the same time, there are numerous examples in which forcing real (non-ideal) physical systems causes them to become knotted: vortices in classical or superfluid turbulence ${ }^{15,16}$, magnetic fields in the solar corona ${ }^{4}$, and defects in condensed matter phases ${ }^{10}$. This presents a conundrum: why doesn't everything get stuck in a tangled web, much like headphone cords in a pocket ${ }^{1}$ ?

In all of these systems, 'reconnection events' allow fields to untangle by cutting and splicing together nearby lines/structures (Fig. 1a; refs 3,4,8-11). As a result, the balance of knottedness, and its fundamental role as a constraint on the evolution of physical systems, depends critically on understanding if and how these mechanisms cause knots to untie.

Previous studies of the evolution of knotted fields have been restricted to relatively simple topologies or idealized dynamics ${ }^{3,9,17,18}$.
Here, we report on a systematic study of the behaviour of all prime topologies up to nine crossings by simulating isolated quantum vortex knots in the Gross-Pitaevskii equation (GPE, equation (1)). The quantum counterpart of smoke rings in air, vortices in superfluids or superconductors are line-like phase defects in the quantum order parameter, $\psi(\mathbf{x})=\sqrt{\rho(\mathbf{x})} \mathrm{e}^{i \phi(\mathbf{x})}$, where $\rho$ and $\phi$ are the spatially varying density and phase (Fig. 1e). The GPE is a useful model system for studying topological vortex dynamics: vortex lines are easily identified, reconnections occur without divergences in physical quantities, and the behaviour of simple knots was recently shown to be comparable to viscous fluid experiments ${ }^{12}$.

In a non-dimensional form, the Gross-Pitaevskii equation is given by ${ }^{19}$ :

$$
\frac{\mathrm{d} \psi}{\mathrm{d} t}=-\frac{i}{2}\left[\nabla^{2}-\left(|\psi|^{2}-1\right)\right] \psi
$$

where in these units the quantized circulation around a single vortex line is given by: $\Gamma=\oint \mathrm{d} \boldsymbol{\ell} \cdot \mathbf{u}=2 \pi$. The GPE has a characteristic length scale, known as the 'healing length', $\xi$, which corresponds to the size of the density-depleted region around each vortex core $(\xi=1$ in our non-dimensional units if the background density is $\left.\rho_{0}=1\right)$.

Producing a knotted vortex in a superfluid model requires the computation of a space-filling complex function whose phase field contains a knotted defect. This challenging step has restricted previous studies to one family of knots in a specific geometry ${ }^{8}$. By numerically integrating the flow field of a classical fluid vortex, we produce phase fields with defects (vortices) of any topology or geometry $^{12}$ (Fig. 1e and Supplementary Movie 1), enabling us to study the evolution of every prime knot and link with nine or fewer crossings, $n \leq 9$.

To construct initial shapes for the different topologies, we begin with the 'ideal' form for each knot, equivalent to the shape of the shortest knot tied in a rope of thickness $r_{0}$ (Fig. 1b-d; ref. 20). These canonical shapes are known to capture key aspects of the knot type as well as approximating the average properties of random knots ${ }^{21}$. For each ideal shape we consider three different overall scalings with respect to the healing length: $r_{0} / \xi=\{15,25,50\}$. To break any symmetries of the shape and to check for robustness of our results we also consider four randomly distorted versions of each knot with $n \leq 8$ at a scale of $r_{0}=15 \xi$ (see Methods for a detailed description of the construction).

Figure 2a and Supplementary Movie 2 show the evolution of a 6-crossing knot, K6-2, as it unties. (We label links and knots using a generalized notation following the 'Knot Atlas', http://katlas.org.) The knot can be seen to deform towards a series of vortex reconnections that progressively simplify the knot until only unknotted rings (unknots) remain. This behaviour has previously been observed for a handful of simple knots and links; here we find the same behaviour in all of the 1,458 simulated vortex knots.

\footnotetext{
${ }^{1}$ James Franck Institute and Department of Physics, The University of Chicago, Chicago, Illinois 60637, USA. ${ }^{2}$ Department of Mathematics, Statistics and Computer Science, University of Illinois at Chicago, Chicago, Illinois 60607, USA. †Present address: University of California, Merced, Merced, California 95343, USA. *e-mail: dkleckner@ucmerced.edu; wtmirvine@uchicago.edu
} 
a

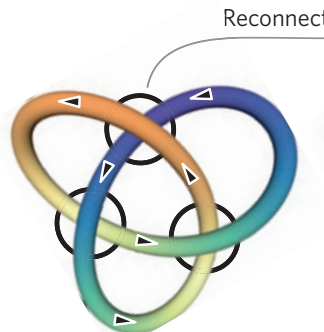

d

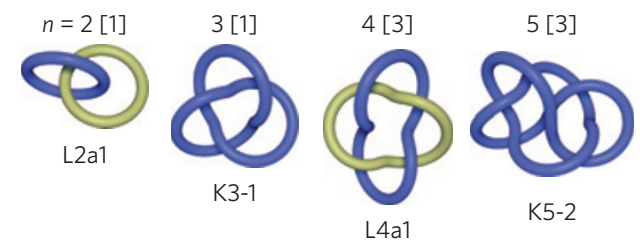

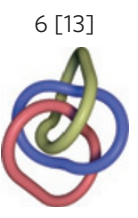

L6n1

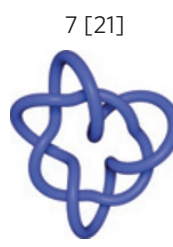

K7-1

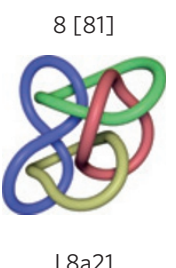

L8a21
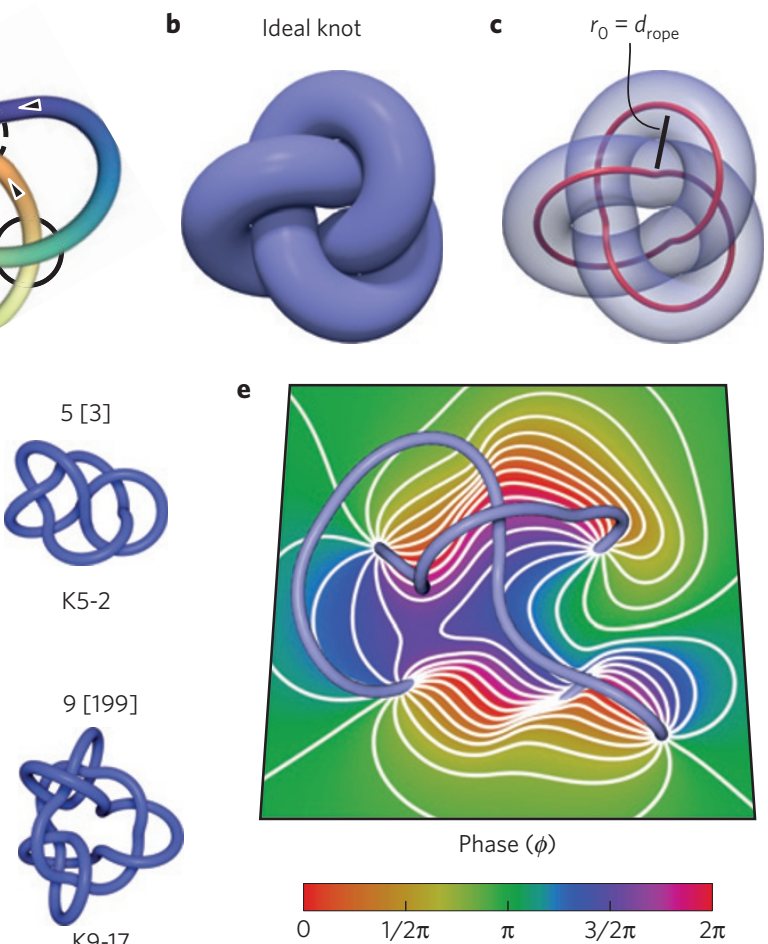

e

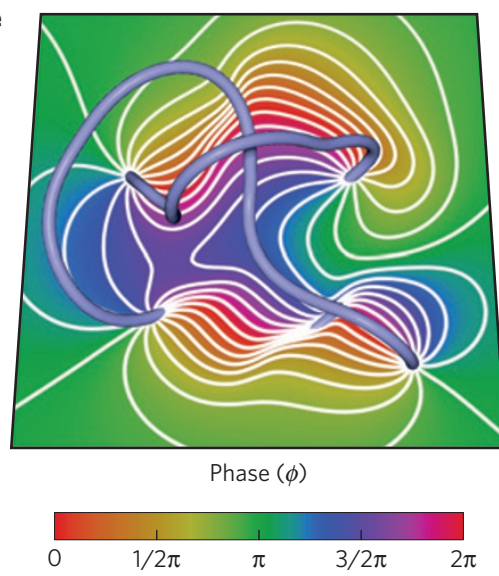

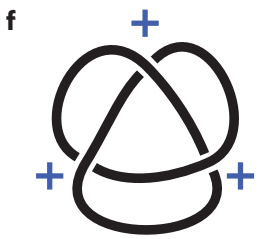

K3-1

$n=3, w=+3$

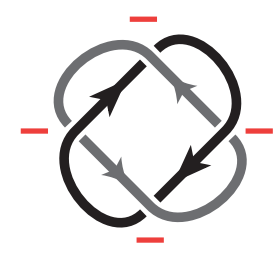

$\mathrm{L} 4 \mathrm{a} 1$

$n=4, w=-4$

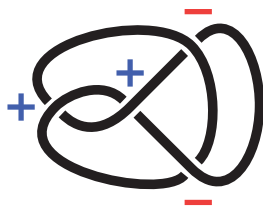

K4-1

$n=4, w=0$

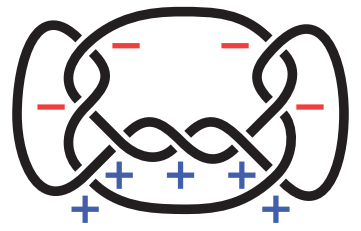

K9-17

$n=9, w=+1$

Figure 1 | Reconnection events and vortex knots. a, A schematic of a vortex reconnection event, in this case converting a trefoil knot (K3-1) to a pair of linked rings (L2a1). b, An 'ideal', or minimum rope-length, trefoil knot. c, Using the centreline of an ideal knot provides a consistent, uniform geometry for any knot or link; nearby strands are exactly spaced by the rope diameter, $d_{\text {rope, }}$ which becomes the characteristic radius, $r_{0}$, of the loops which compose the knot. d, Example ideal configurations of topologies with different minimal crossing number, $n$. The number of topologies excluding mirrored pairs is indicated in square brackets. e, A 2D slice of the phase field of a superfluid order parameter with a knotted vortex line (light blue). f, Example minimal knot diagrams; in each case the topology cannot be represented by a simpler planar diagram. The chirality of each crossing is indicated.

We further note that during the evolution of any sufficiently complex knot, strongly distorted forms of simpler vortex knots are produced, which all in turn exhibit similar untying dynamics to their more ideally shaped counterparts.

We quantify the vortex dynamics by computing the dimensionless length, vortex energy and helicity, as a function of time (Fig. 2c-e and Supplementary Fig. 2, see Methods for details). The vortex energy, computed from the shape of the superfluid phase defect, measures the energy associated with the vortical flow, as opposed to sound waves. The total combined energy (from vortices and sound waves) is conserved in the GPE unless a dissipative term is added; we do not include one here.

The non-dimensional 'centreline helicity', $h$ - which measures the total linking, knotting and coiling in the field—is given by ${ }^{6,7,12,22}$ :

$$
h=\sum_{i \neq j} L k_{i j}+\sum_{i} W r_{i}
$$

where $L k_{i j}$ is the linking number between vortex lines $i$ and $j$, and $W r_{i}$ is the $3 \mathrm{D}$ writhe of line $i$, which includes contributions from knotting as well as helical coils. Note that helicity in a classical fluid would include a term proportional to the twist inside the core (see Methods for a discussion of twist in the context of superfluid cores).

Three general trends can be clearly discerned from our results: the timescale for unknotting is determined predominantly by the overall scale of the knot, $r_{0} / \xi$, where $r_{0}$ is the rope thickness of the ideal shape used to generate the initial state (Fig. 3a-d); the helicity is not simply dissipated, but rather converted from links and knots into helical coils, with an efficiency that depends on scale (Fig. $3 \mathrm{e}-\mathrm{h}$ ); and the vortex lines stretch by $\sim 20 \%$ as they untie, even though the vortex energy decreases slightly. We note that the vortex energy changes through reconnections, as some energy is converted to sound waves (in line with previous observations of colliding rings ${ }^{23}$ ). Interestingly, all of these results are apparently, on average, independent of knot complexity: for the same scale, $r_{0}$, simple knots untie just as quickly as complicated ones, and lose the same relative amount of helicity and vortex energy (Supplementary Fig. 3). We furthermore note that these results are also consistent with previous results for knots in experimental viscous fluids and Biot-Savart simulations ${ }^{12,24}$.

Conversion of helicity from knots and links to helical coils has previously been observed for trefoil knots and linked rings 


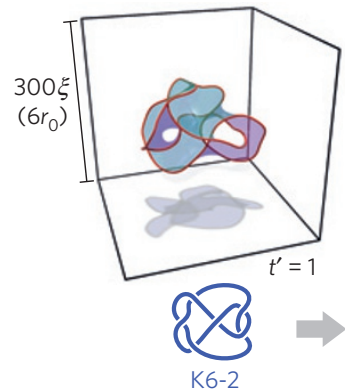

b

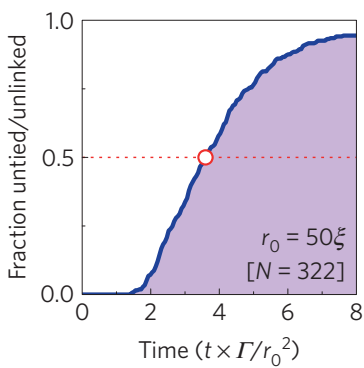

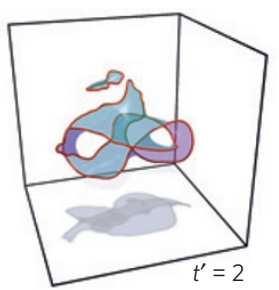

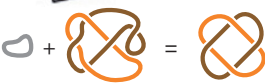

L4a1

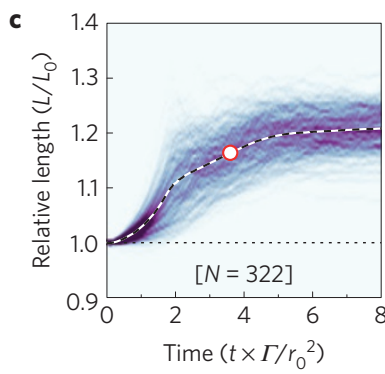

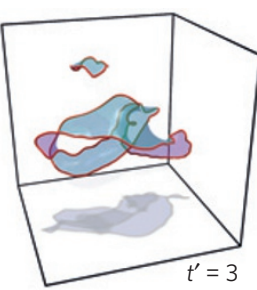

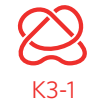

d
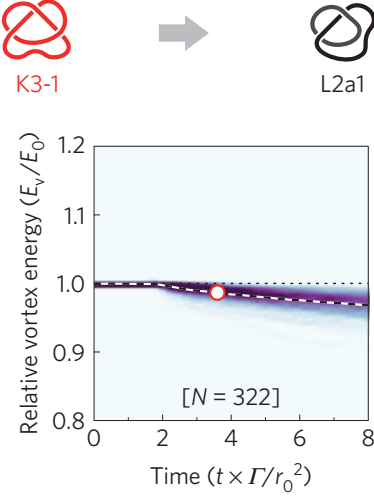

L2a1
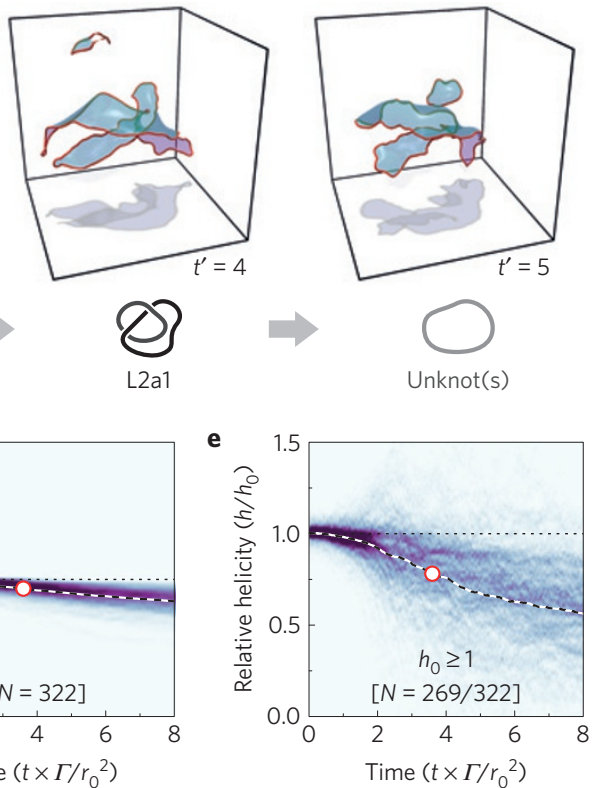

Figure 2 | Geometric evolution of vortex knots. a, The untying of a randomly distorted 6 -crossing knot (K6-2, $\left.r_{0}=50 \xi\right)$ to a collection of unknotted rings. The rescaled time, $t^{\prime}=t \times \Gamma / r_{0}{ }^{2}$, is shown for each step. The top section shows density iso-surfaces of the local order parameter (red, $\left.|\psi|^{2}=1 / 2\right)$ and the transparent surfaces (teal or purple) show a constant phase iso-surface. Each volume has been centred on the vortex, which would otherwise have a net vertical motion; only $48 \%$ of the simulation volume is shown. $\mathbf{b}$, The fraction of simulations that have untied/unlinked as a function of time, computed for the 322 simulations of ideal knots with $r_{0}=50 \xi$. The median unknotting time is indicated in red. c-e, $2 \mathrm{D}$ histograms of relative length, vortex energy and helicity as a function of time for all prime topologies with $n \leq 9$. The dashed lines indicate average values. The helicity histogram (e) includes only the $269 / 322$ topologies with $h_{0} \geq 1$. See Supplementary Fig. 2 for similar histograms for each group of simulations.

a
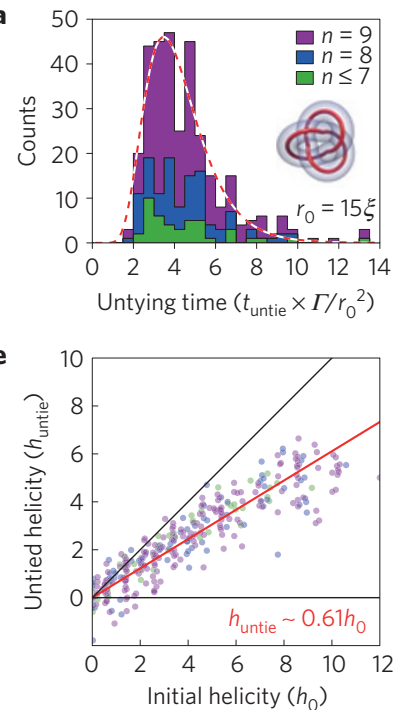

b
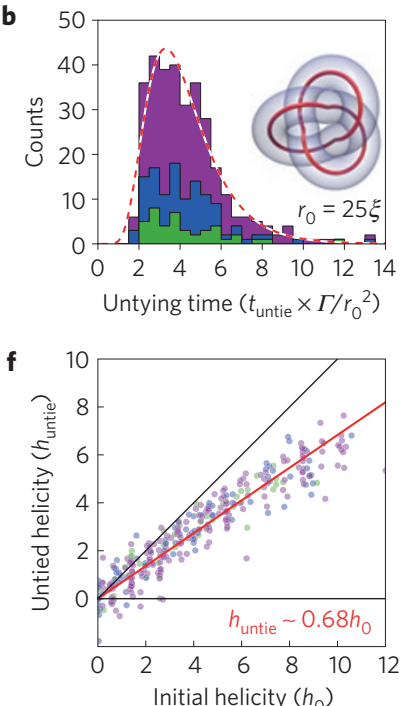

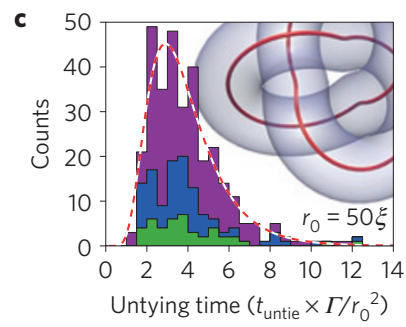

g

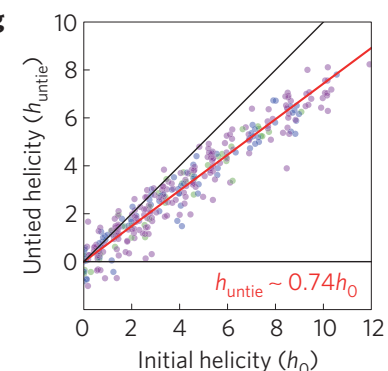

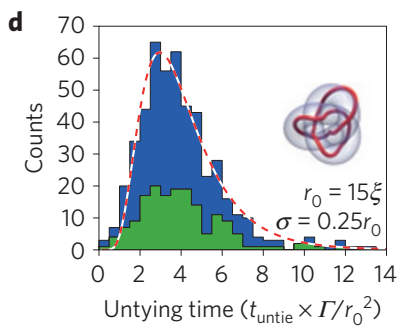

h

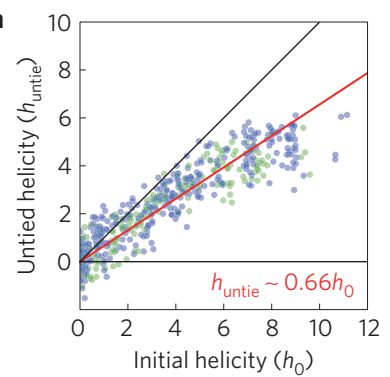

Figure 3 | Statistics of vortex knot untying. Histograms of the rescaled untying time (a-d) and the untied versus initial helicity (e-h) for four different groups of simulations: $\mathbf{a}-\mathbf{c}, \mathbf{e}-\mathbf{g}$, All 322 ideal knots with $n \leq 9$ at a scale of $r_{0}=\{15,25,50\} \xi$. $\mathbf{d}, \mathbf{h}$, Four randomly distorted versions of each $n \leq 8$ ideal knot

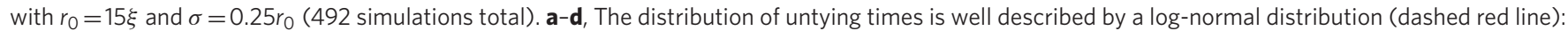
$P\left(t^{\prime}\right) \propto\left(1 / t^{\prime}\right) \exp \left[-\left(\left(\ln t^{\prime}-\mu\right)^{2} /\left(2 \sigma^{2}\right)\right)\right]$, where the average unknotting time is $\left\langle t^{\prime}\right\rangle \approx \exp \mu=\{4.0,3.9,3.5,3.7\}$ and the spread is $\sigma=\{0.37,0.41,0.44,0.47\}$ for $\mathbf{a}$-d respectively. $\mathbf{e}-\mathbf{h}$, The final helicity is approximately proportional to the initial helicity (red line). The degree to which helicity is preserved depends on overall scale, but is apparently only slightly affected by randomly distorting the knots. (This slight difference might be explained by the knots being effectively larger from the distortion.)

in classical fluids ${ }^{12}$, and can be explained through a geometric mechanism. After each reconnection event, helices with a range of length scales are produced on the reconnected vortices. If one assumes a perfectly antiparallel reconnection without any spatial cutoff, this process is expected to exactly conserve helicity ${ }^{12,25}$. However, in the GPE, helical distortions on the scale of the healing length are radiated away as sound waves (Supplementary Movie 4). As a result, we observe an average helicity loss with an approximate $\Delta h / h_{0} \propto\left(r_{0} / \xi\right)^{-0.5}$ trend. Remarkably, these results suggest that as the scale becomes very large, $r_{0} \gg \xi$, helicity conservation should be recovered even though knots still untie. 
a
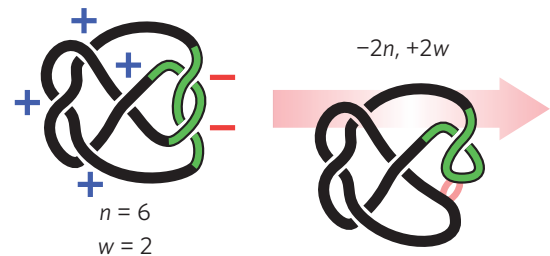

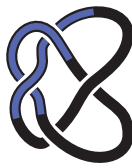

$n=4$

$w=4$

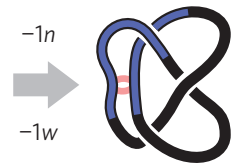

$n=3$

$w=3$

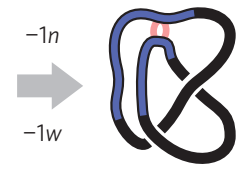

$n=2$

$w=2$

b

Relaxation of antiparallel pair

C

Relaxation of parallel pair
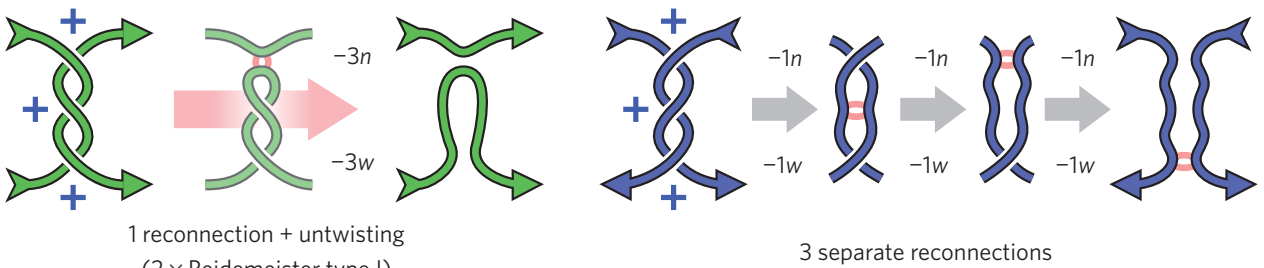

$(2 \times$ Reidemeister type I)

3 separate reconnections

Figure 4 | Topological mechanisms for untying vortex knots. a, Knot diagrams of an observed decay pathway for a 6-crossing knot; all steps can be described by local untwisting events. The minimal crossing number, $n$, and topological writhe, $w$, is labelled for each diagram. $\mathbf{b}, \mathbf{c}$, Nearly all reconnection events can be classified either as relaxation of a twisted pair in an antiparallel or parallel orientation; in either case $\Delta n=-|\Delta w|$. Reconnections of antiparallel pairs are equivalent to a crossing removal plus one or more type-I Reidemeister moves.

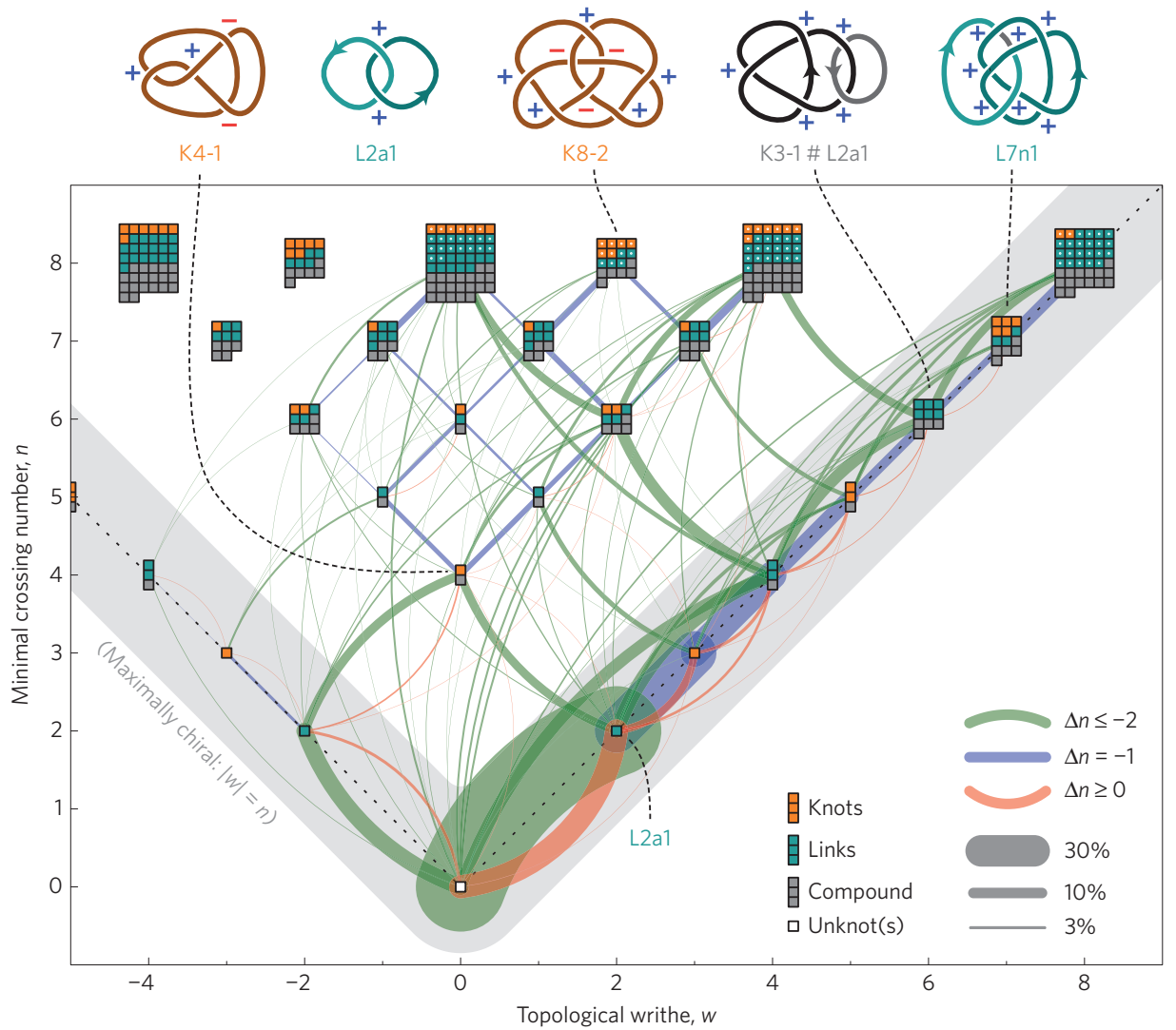

Figure $\mathbf{5}$ | Unknotting pathways in terms of minimal crossing number, $\boldsymbol{n}$, and topological writhe, $\boldsymbol{w}$. The squares indicate the total number of topologies at each point, including non-prime links/knots; five example topologies are shown on top. Four randomly distorted copies of each $n=8$ prime link/knot are used as the starting points (marked with white dots; only one handedness of chiral topologies is considered). Green and blue lines indicate reconnections which reduce the crossing number, whereas red lines show events which increase crossing number. The lightly shaded region indicates the maximally chiral topologies.

If one assumes that concentrated vorticity distributions will always expand, the observation that knots universally untie has an intuitive description. Collections of unknotted vortex rings may separate without stretching individual vortex lines, but a linked or knotted structure must stretch to expand. At the same time, if the system is undriven the vortex lines must reorient to conserve energy as they stretch: as previously observed for simple knots, the formation of closely spaced, antiparallel vortex pairs reduces the energy per unit length ${ }^{3}$. As the stretching continues, these antiparallel regions are driven closer together until they 
ultimately reconnect; this process continues until the knots are completely untied. We note that, in most cases, the stretching stops abruptly after the knots finish untying (Supplementary Fig. 1), in agreement with this interpretation. Interestingly, such a picture also naturally produces the antiparallel reconnection geometry that favours helicity conservation.

Although the above results demonstrate the overwhelming tendency for vortex knots to untie, they do not elucidate the specific topological pathways which produce this untangling. To measure these unknotting sequences, we identify the topology, $T_{i}$, of the vortices after each reconnection by computing their HOMFLYPT polynomials ${ }^{26,27}$. Owing to the high degree of symmetry of ideal knots, reconnections are often nearly coincident in time, preventing identification of the intermediate topology. To avoid this complication, we consider only the decays of the randomly distorted knots, which break this symmetry.

The first question we examine is whether the knot is simplifying at each step. We quantify the knot complexity by means of the crossing number, $n$, of each knot in a minimal two-dimensional (2D) diagram (Fig. 1f), which is a topological invariant of the knot. Supplementary Table 2 shows the statistics of the jumps in the crossing number through all reconnections, revealing knots are about an order of magnitude more likely to 'untie' $(\Delta n<0)$ than 'retie' $(\Delta n>0)$ at each individual reconnection. On average, more than one crossing is removed with each reconnection, underscoring the fact that physical reconnections of vortices in $3 \mathrm{D}$ are not equivalent to removing (or adding) a single crossing from a $2 \mathrm{D}$ minimal knot diagram. Nonetheless, the minimal diagrams reveal a clear trend towards topological simplification.

If each reconnection does not correspond to 'removing' a single crossing from a $2 \mathrm{D}$ knot diagram, is it still possible to produce an intuitive description of these events in terms of such diagrams? This question can be answered by considering the $2 \mathrm{D}$ topological writhe, $w\left(T_{i}\right)$, which is obtained by summing the handedness $( \pm 1)$ of each crossing in a minimal knot diagram (obtained from ref. 28). Remarkably, we find that the vast majority (96.1\%) of reconnection events only add/remove crossings of the same sign from 2D diagrams, that is, $|\Delta n|=|\Delta w|$ (including events with remove a single crossing). As shown in Fig. $4 b, c$, reconnections which satisfy this condition are equivalent to the relaxation of a parallel or antiparallel pair in a 2D diagram. For the perspective of minimal diagrams, the removal of multiple crossings occurs by a single reconnection in an antiparallel pair, followed by the untwisting of a topologically trivial loop by type-I Reidemeister moves ${ }^{29,30}$ (Fig. 4b). Reconnections followed by more complicated simplifications are possible (for example, incorporating type-II Reidemeister moves); however, such events are observed to be rare.

Figure 5 and Supplementary Movie 5 show the topological writhe and crossing number of every knot with $n \leq 8$, including nonprime topologies, connected by lines indicating the frequency of the observed unknotting pathways. (This is similar to diagrams which have previously been constructed for mathematical knot simplification of a different type ${ }^{31}$.) In addition to illustrating the above results, this diagram reveals the importance of the 'maximally chiral' topologies, for which $|w|=n$. The topological writhe for any particular knot or link is bounded by the number of crossings; maximally chiral knots and links saturate this bound, which corresponds to every crossing having the same sign.

Despite the fact that only around a third of all $n \leq 8$ topologies are maximally chiral, $82.6 \%$ of jumps end in such a state. The dominance of this pathway has a simple interpretation: if we assume all reconnections satisfy $|\Delta n| \geq|\Delta w|$, corresponding to a slope of $|\Delta n / \Delta w| \geq 1$ in Fig. 5, once the vortex knot decays into a maximally chiral topology it can leave such a state only by increasing its crossing number. (Although the observation that $|\Delta n| \geq|\Delta w|$ seems self-evident when considering minimal crossing diagrams, we are not aware of a proof of this relationship. Nonetheless, we never observe reconnections which violate it.) Indeed, owing to the 'gap' between maximally and non-maximally chiral states, the crossing number must increase by $\Delta n \geq+2$ to leave the maximally chiral branch. Moreover, even in the event that the crossing number does increase by this amount, we observe that it still typically stays on the maximally chiral branch. Thus, statistically, most knots are funnelled into a maximally chiral pathway during their untying, after which they decay only along this pathway.

Our observation of a preferred maximally chiral pathway is a generalization of a previously known result for site-specific recombination of DNA knots: any $p=2$ torus knot/link (which are all maximally chiral) may convert into another $p=2$ torus knot by means of reconnections only if the crossing number is decreasing ${ }^{2}$. Our results indicate that this torus knot pathway is one example of a more general phenomena. Intuitively, this suggests untangling knots tend to end up in states which are twisted in only one chiral direction.

Taken as a whole, we find that the topological behaviour of superfluid vortex knots and links can be understood through simple principles. All vortex knots untie, and they tend to do so efficiently: monotonically decreasing their crossing number until they are a collection of unknotted vortices. This suggests that non-trivial vortex topology in superfluids-or any fluid with similar topological dynamics-should arise only from external driving. Even in the presence of driving, the observed decay pathways indicate that vortices would probably settle into a maximally chiral topology; it would be of great interest to probe for such states in superfluid or classical turbulence.

The evolution and untying dynamics of the superfluid knots we observe is strongly reminiscent of those in classical fluids and DNA recombination ${ }^{2,3,12}$. These similarities persist despite fundamental differences between these systems, especially with regards to the small-scale details of the reconnection processes that drive topology changes. This suggests that they might apply even more generally, forming a universal set of mechanisms for understanding the evolution of knots in a variety of dissipative physical systems.

\section{Methods}

Methods and any associated references are available in the online version of the paper.

Received 23 September 2015; accepted 25 January 2016; published online 7 March 2016

\section{References}

1. Raymer, D. M. \& Smith, D. E. Spontaneous knotting of an agitated string. Proc Natl Acad. Sci. USA 104, 16432-16437 (2007).

2. Shimokawa, K., Ishihara, K., Grainge, I., Sherratt, D. J. \& Vazquez, M. FtsK-dependent XerCD-dif recombination unlinks replication catenanes in a stepwise manner. Proc. Natl Acad. Sci. USA 110, 20906-20911 (2013).

3. Kleckner, D. \& Irvine, W. T. M. Creation and dynamics of knotted vortices. Nature Phys. 9, 253-258 (2013).

4. Cirtain, J. W. et al. Energy release in the solar corona from spatially resolved magnetic braids. Nature 493, 501-503 (2013).

5. Thomson, W. On vortex atoms. Philos. Mag. XXXIV, 94-105 (1867).

6. Moffatt, H. K. Degree of knottedness of tangled vortex lines. J. Fluid Mech. 35, 117-129 (1969)

7. Berger, M. A. Introduction to magnetic helicity. Plasma Phys. Control. Fusion 41, B167-B175 (1999)

8. Proment, D., Onorato, M. \& Barenghi, C. Vortex knots in a Bose-Einstein condensate. Phys. Rev. E 85, 1-8 (2012).

9. Wasserman, S. A. \& Cozzarelli, N. R. Biochemical topology: applications to DNA recombination and replication. Science 232, 951-960 (1986).

10. Tkalec, U. et al. Reconfigurable knots and links in chiral nematic colloids. Science 333, 62-65 (2011). 
11. Bewley, G. P., Paoletti, M. S., Sreenivasan, K. R. \& Lathrop, D. P. Characterization of reconnecting vortices in superfluid helium. Proc. Natl Acad. Sci. USA 105, 13707-13710 (2008).

12. Scheeler, M. W., Kleckner, D., Proment, D., Kindlmann, G. L. \& Irvine, W. T. M. Helicity conservation by flow across scales in reconnecting vortex links and knots. Proc. Natl Acad. Sci. USA 111, 15350-15355 (2014).

13. Sumners, D. Lifting the curtain: using topology to probe the hidden action of enzymes. Not. Am. Math. Soc. 528-537 (1995).

14. Woltjer, L. A theorem on force-free magnetic fields. Proc. Natl Acad. Sci. USA 44, 489-491 (1958).

15. Moffatt, H. \& Ricca, R. Helicity and the Calugareanu invariant. Proc. R. Soc. Lond. A 439, 411-429 (1992)

16. Barenghi, C. F. Knots and unknots in superfluid turbulence. Milan J. Math. 75, 177-196 (2007)

17. Dennis, M. R., King, R. P., Jack, B., O’holleran, K. \& Padgett, M. Isolated optical vortex knots. Nature Phys. 6, 118-121 (2010).

18. Martinez, A. et al. Mutually tangled colloidal knots and induced defect loops in nematic fields. Nature Mater. 13, 258-263 (2014).

19. Pitaevskii, L. P. \& Stringari, S. Bose-Einstein Condensation (Clarendon, 2003)

20. Pieranski, P. in Ideal Knots (eds Stasiak, A., Katritch, V. \& Kauffman, L. H.) (World Scientific, 1998).

21. Katritch, V. et al. Geometry and physics of knots. Nature 384, 142-145 (1996).

22. Akhmet'ev, P. \& Ruzmaikin, A. in Topological Aspects of the Dynamics of Fluids and Plasmas Vol. 218 (eds Moffatt, H. K., Zaslavsky, G. M., Comte, P. \& Tabor, M.) 249-264 (NATO ASI Series, Springer, 1992).

23. Leadbeater, M., Winiecki, T., Samuels, D. C., Barenghi, C. F. \& Adams, C. S. Sound emission due to superfluid vortex reconnections. Phys. Rev. Lett. 86, 1410-1413 (2001).

24. Ricca, R. L., Samuels, D. \& Barenghi, C. Evolution of vortex knots. J. Fluid Mech. 391, 29-44 (1999).

25. Laing, C. E., Ricca, R. L. \& Sumners, D. W. L. Conservation of writhe helicity under anti-parallel reconnection. Sci. Rep. 5, 9224 (2015).

26. Freyd, P. et al. A new polynomial invariant of knots and links. Bull. Am. Math. Soc. 12, 239-246 (1985).
27. Przytycki, J. H. \& Traczyk, P. Conway algebras and skein equivalence of links. Proc. Am. Math. Soc. 100, 744-748 (1987).

28. Cha, J. C. \& Livingston, C. Knot Info: Tables of Knot Invariants (February 2015); http://www.indiana.edu/ knotinfo

29. Reidemeister, K. Abhandlungen aus dem Mathematischen Seminar der Universität Hamburg Vol. 5, 24-32 (Springer, 1927).

30. Alexander, J. W. \& Briggs, G. B. On types of knotted curves. Ann. Math. 28, 562-586 (1926)

31. Flammini, A. \& Stasiak, A. Natural classification of knots. Proc. R. Soc. Lond. A 463, 569-582 (2007).

\section{Acknowledgements}

The authors acknowledge M. Scheeler and D. Proment for useful discussions. This work was supported by the National Science Foundation (NSF) Faculty Early Career Development (CAREER) Program (DMR-1351506), and completed in part with resources provided by the University of Chicago Research Computing Center and the NVIDIA Corporation. W.T.M.I. further acknowledges support from the A.P. Sloan Foundation through a Sloan fellowship, and the Packard Foundation through a Packard fellowship.

\section{Author contributions}

D.K. and W.T.M.I. designed and developed the study. D.K. performed simulations and knot identification. D.K., L.H.K. and W.T.M.I. analysed data. D.K. and W.T.M.I. wrote the manuscript.

\section{Additional information}

Supplementary information is available in the online version of the paper. Reprints and permissions information is available online at www.nature.com/reprints. Correspondence and requests for materials should be addressed to D.K. or W.T.M.I.

\section{Competing financial interests}

The authors declare no competing financial interests. 


\section{Methods}

Simulation details. The time evolution of the superfluid order parameter was computed by numerically integrating the Gross-Pitaevskii equation using a split-step spectral method along the lines of refs 8,12 . For simulations with mean radius $r_{0} / \xi=\{15,25\}$, we use a grid size of $\Delta x=0.5 \xi$, a simulation time step of $\Delta t=0.02$, and save the traced vortex paths at an interval of $\Delta T=1$. For simulations with $r_{0}=50 \xi$, we compute a coarser simulation with $\Delta x=1 \xi, \Delta t=0.1$ and $\Delta T=4$. The radial distance from the vortices at which the order parameter, $|\psi|$, recovers to half its far-field value (often referred to as the 'core size') is determined by the healing length and is approximately $R \sim 2 \xi$. A small number of simulations of the same sized knot at different resolutions $(\Delta x / \xi=\{0.25,0.5,1\})$ was used to confirm that the coarser simulations do not significantly affect the computed length and helicity of the vortex as it unties (the noise in these computed quantities does increase, but we do not observe systematic differences). The total size of the periodic simulation box was $L / \xi=\{128,192,384\}$ for $r_{0} / \xi=\{15,25,50\}$, respectively. Occasionally, small vortex rings ejected from the untying vortices interact with their periodic partners by traversing the boundary; in general this happens only after the vortices have untied. To ensure that the size of the box does not affect the behaviour of the knot, we have simulated the same knot in several differently sized periodic volumes; we find that the behaviour of the knot is virtually identical so long as it is spaced more than a few $r_{0}$ from its periodic partner (in practice, the most complex knots have a maximum extent of only around half the length of edge of the simulation box).

We note that we employ a version of the GPE without dissipation, and so the total energy is conserved. Numerically, there is some small loss (always less than $1 \%$ and typically less than $0.2 \%$ ), which is not significant for any of our results. We also include a chemical potential of $\mu=-1$ (in dimensionless units) in our definition of the GPE; this is added to remove an overall phase rotation. Removing this additional term would produce mathematically identical results, as the overall phase is not physically significant.

Initial state construction. The phase fields for the initial states were generated by brute force integration of a Biot-Savart-generated flow field, $\mathbf{u}_{\mathrm{BS}}$, which is related to the phase gradient through the relationship:

$$
\nabla \phi(\mathbf{x})=\mathbf{u}_{\mathrm{BS}}(\mathbf{x})
$$

This method is described in more detail in ref. 12. An example an initial phase field is shown in Fig. 1e and Supplementary Movie 1.

The initial density field, $\rho=|\psi|^{2}$, was calculated using an approximate form obtained for an infinite, straight vortex line ${ }^{32}$ :

$$
\rho(r)=\frac{\frac{11}{32} r^{2}+\frac{11}{384} r^{4}}{1+\frac{1}{3} r^{2}+\frac{11}{384} r^{4}}
$$

where $r$ was taken to be the distance to the closest vortex line.

To ensure consistency between different topologies, we choose the 'ideal' form of each knot, equivalent to the shape of the shortest knot tied in a finite thickness rope (Fig. 1b-d; ref. 20). These canonical shapes are known to capture aspects of the knot type as well as approximating the average properties of random knots ${ }^{21}$, making them a useful reference geometry for each topology. The shapes of ideal knots for different topologies were obtained from an online source

(http://katlas.math.toronto.edu/wiki/Ideal_knots), and were initially generated by means of the SONO method ${ }^{20}$.

To create randomly distorted knots, we compute a random normally distributed vector, $\delta$, for each point in a polygonal representation of the vortex knot in question. We then smooth this vector with a Gaussian of width $\sigma=0.5 r_{0}$ (measured along the path of original vortex line), remove the component tangential to the original knot path, and rescale the displacement vector so that $\left\langle|\delta|^{2}\right\rangle=\left(0.25 r_{0}\right)^{2}$. This displacement is added to the original coordinates to obtain the distorted knot. Examples of randomly distorted knots are shown in Fig. 2a and the inset of Fig. $3 \mathrm{~d}$. For the data shown in the paper, four randomly perturbed copies of each $n \leq 8$ knot/link ( $4 \times 123$ configurations) were considered at a scale of $r_{0}=15 \xi$. A small number of randomly distorted knots at larger scales were considered, producing qualitatively similar effects to the scaling of undistorted ideal knots.

Quantification of vortex behaviour. For each saved time step, a polygonal representation of the vortex shape is obtained by tracing the phase defects in the superfluid order parameter with a resolution set by the simulation grid (typically resulting in $\gtrsim 10^{3}$ points total). In addition, a phase normal, $\hat{\phi}$, is computed for each point on the vortex by finding the direction of zero phase which is perpendicular to the vortex path. All subsequent properties (vortex energy, helicity, and length) are computed from this path.

To determine the moment which a knot finishes untying, we find the moment at which its HOMFLY-PT polynomial is equivalent to unknots (see 'Identification of Vortex Topology' below). A histogram of the unknotting times, Fig. 3a-d, is consistent with a log-normal distribution. We find that once the time is appropriately rescaled, the mean unknotting times for each simulation group are in the range $\left\langle t_{\text {unknot }}\right\rangle \approx(3.5-4.0) r_{0}{ }^{2} / \Gamma\left(r_{0}\right.$ is the diameter of the 'rope' in which the ideal knot is tied).

As stated in the main text, we compute the centreline helicity in the dimensionless form:

$$
h=\sum_{i \neq j} L k_{i j}+\sum_{i} W r_{i}
$$

Although this can be computed directly from the polygonal paths, this method requires special considerations for dealing with linking across periodic boundaries. Alternatively, we may note that a surface of constant phase defines a 'Seifert framing' for each knot/link obeying $h+\sum_{i} T w_{\phi, i}=0$ (ref. 22), where $T w_{\phi, i}$ is the twist of the phase normal about the vortex path:

$$
T w_{\phi, i}=\oint_{\mathcal{C}_{i}} \mathrm{~d} \ell \cdot \hat{\phi} \times \partial_{s} \hat{\phi}
$$

where $\mathcal{C}_{i}$ refers to closed vortex path $i, \partial_{s}$ is a path-length derivative and $\hat{\phi}$ is a unit vector that is lies along a surface of equal phase and is perpendicular to the tangent vector of the vortex line at that point. As the total twist can be easily numerically integrated from the vortex path and phase normal, it provides an efficient method for computing centreline helicity. We have numerically confirmed that this method provides results equal to direct computation of linking and writhe, up to numerical precision.

The energy associated with the vortices in the superfluid (as opposed to sound waves), $E_{\mathrm{v}}$, is computed from the 'path inductance ${ }^{3}, \mathcal{E}_{i j}$, of the vortex centrelines:

$$
\begin{gathered}
E_{\mathrm{v}} \cong \frac{\rho \Gamma^{2}}{2} \sum_{i j} \mathcal{E}_{i j} \\
\mathcal{E}_{i j}=\frac{1}{4 \pi} \oint_{\mathcal{C}_{i}} \oint_{\mathcal{C}_{j}}^{\left|\mathbf{r}_{i}-\mathbf{r}_{j}\right|>\frac{\xi}{2}} \frac{\mathrm{d} \mathbf{r}_{i} \cdot \mathrm{d} \mathbf{r}_{j}}{\left|\mathbf{r}-\mathbf{r}_{j}\right|}+\delta_{i j} \frac{2-\alpha}{2 \pi} L_{i}
\end{gathered}
$$

where $L_{i}$ is the total length of vortex loop $i$.

Here, $\alpha \cong 1.615$ is a dimensionless correction factor chosen to obtain the correct value for the energy of a vortex ring in the GPE (ref. 33). To account for the periodic nature of the simulations, the cross inductance is included for a $3 \times 3 \times 3$ periodic array of vortex paths (including more periodic copies improves the accuracy of the calculation, but the difference is typically only a small fraction of a per cent). Note that the total energy in the superfluid is conserved, so a reduction in vortex energy corresponds to an increase in the energy in sound waves. The vast majority of energy changes in the vortices are seen to occur during or immediately after reconnection events; otherwise the computed energy is nearly constant.

Identification of vortex topology. To identify the topology of the superfluid vortices at each time step, the polygonal vortex representation is first reduced to the minimum possible number of points possible without changing the topology (unknots are also removed at this stage, if they are not threaded by any other vortex lines). Once the vortices are reduced, they are projected into an arbitrary $2 \mathrm{D}$ plane and the projected crossings and their handedness are identified; the HOMFLY-PT polynomial is created directly from this crossing list. This polynomial is compared against an internally generated database of HOMFLY-PT polynomials for all topologies (including chiral pairs, oriented links, and disjoint and compound knots/links) with a minimal crossing number of $n \leq 10$. As mentioned in the main text, we label topologies following the format used by the 'Knot Atlas', http://katlas.org, for example, a 'stevedore's knot' is $K 6-1$, with the ' $K$ ' indicating it is a knot (versus a link, 'L'), $n=6$ is the minimal crossing number (Fig. 1f), and the remainder indicates an arbitrary ordering.

The database of HOMFLY-PT polynomials for prime topologies was generated starting from the crossing diagrams obtained from ref. 28. The equivalence of oriented links was determined by assuming all orientational permutations with identical HOMFLY-PT polynomials are topologically equivalent. The HOMFLY-PT polynomial of disjoint and compound topologies was computed algebraically from the HOMFLY-PT polynomials of their components, and added to the list. We do not treat configurations with extra unknots to be distinct topologies, and do not distinguish between disjoint and compound topologies. (We note that disjoint knots are rarely observed in the decay pathways, and are furthermore difficult to distinguish from compound knots by means of HOMFLY-PT polynomials if unknots are also present.) There exist several knots/links with identical HOMFLYPT polynomials for $n \geq 9$, but we do not encounter any of these in the observed pathways for knots starting with $n \leq 8$, which were used to compute decay pathways.

\section{References}

32. Berloff, N. G. Padé approximations of solitary wave solutions of the Gross-Pitaevskii equation. J. Phys. A 37, 1617-1632 (2004).

33. Donnelly, R. J. Vortex rings in classical and quantum systems. Fluid Dyn. Res. 41, 051401 1-31 (2009). 